\title{
Hidden circulation fluctuations periodicity of index daily return
}

\author{
Yuanyuan Ma \\ Northeastern University \\ Shenyang, P. R of China \\ e-mail: mayuanyuan@mail.neu.edu.cn
}

\author{
Lingxuan $\mathrm{Li}$ \\ Northeastern University \\ Shenyang, P. R of China \\ e-mail: lingxuan@mail.neu.edu.cn
}

\begin{abstract}
In this article, we collect the index daily return to analysis the fluctuations of daily return ratio and monthly return ratio in the stock markets by using the multisignal classification (MUSIC) method of Sub-space of the power spectrum density estimation. 6 kinds of Composite Index of stock price are selected, including 3 kinds of index from Shanghai Stock Exchange, Shenzhen Stock Exchange, Hong Kong Stock Exchange, and 3 kinds of index from US Stock Markets, UK stock markets, and Japanese stock markets. Though our research, we get the hidden circulation fluctuations periodicity of index daily return about Chinese stock markets and foreign stock markets. We find that Hang Seng Index has obviously cyclical nature with greatly fluctuation. The Shanghai Composite Index has a similar cyclical nature to London's FTSE 100 index and Shenzhen stock composite prices index, but the London's FTSE 100 index has strength fluctuation. Using the method mentioned in this paper is beneficial to further explore some useful objective phenomena in economic field.
\end{abstract}

Keywords- stock markets; power spectrum density estimation; multi-signal classification(MUSIC); daily return; index of stock price

\section{INTRODUCTION}

The stock market is an open, complex and highly nonlinear dynamic fuzzy stochastic system, the changes of stock price and the fluctuations of return ratio are the typical nonlinear chaotic time series of social economy. Many economic experts and scholars have explored some analysis method which can accurately research some hidden rules of stock market. Analysis shows that the kinematics and dynamics of the fluctuation of return and price in stock markets cover physics, biology, and nonlinear science, vibration theory, sociology, economics and human psychology, etc.[1-7].

I also have research a series of issues about stock markets using different method. We have established the cross-shareholding networks of the listed companies in China to analyze the domestic mutual investment relationships at company-level, province level, and regionlevel[8]. We take the Shanghai Stock Exchange as example to study the characteristics of rich-club in a Chinese stock market[9]. In that paper, we have given out the definition of hub vertices in Chinese stock markets.

In the vibration engineering, a common research method is power spectrum density estimation[10]. It utilizes a certain number of observed sample data to estimate power spectrum density of a stationary random signal. Spectrum analysis, the commonly used method can be divided into classical spectrum estimation and modern spectrum estimation method. Classic spectrum estimation method also can be divided into the direct and indirect method. The direct method uses Discrete Fourier Transformation (DFT) to get the power spectrum method of finite sample data, also called periodogram. The indirect method is to get the autocorrelation function estimates of the sample data, then obtains the power spectrum density by Fourier transforming. Because of a series of disadvantages, such as shaky curve of power spectrum estimation and low resolution of power spectrum, some improved algorithms are put forward, such as BARTLETT method, WELCH method, etc. Their application has extend to many fields, for example, the detection of weak signal in the high noise condition, the fault diagnosis based on analysis of vibration signals, analysis of radar and sonar echo signal, etc.[11-13].

In this paper, we expand the application of power spectral density estimation method to financial markets, and do some explorative research. We also obtain some useful results and reasonable explanation. The whole paper is divided into four sections. The first section is the introduction, the second section is the introducing of the data sources and methods, the third part is the empirical research, and the fourth part is the main conclusion.

\section{DATA AND METHODS}

The data used in this paper is from the RESSET Financial Research Database (www.resset.cn), which mainly contains index daily return. The calculated data is selected from January 1, 2010 to march 30, 2014. In order to verify whether the fluctuation of the Chinese stock markets are the same as the foreign stock markets, 6 indices are selected, including Shanghai Composite Index (Index Code: 000001) from Shanghai Stock Exchange (SSE), Shenzhen stock composite prices index (Index Code: 399106) from Shenzhen Stock Exchange (SZSE), The Hang Seng Index (Index Code: HIS) form Hong Kong Stock Exchange, Nasdaq Composite Index (Index Code: I05031) from US Stock Markets, London's FTSE 100 index (Index Code: I03001) from UK stock markets, Nikkei 225 index (Index Code: I01021) from Japanese stock markets.

\section{METHODS}

The mainly used method in this paper is the MUSIC method of Sub-space of the power spectrum density 
estimation to analysis the fluctuations of index daily return in the stock markets. The Sub-space method is often called high resolution or super-resolution method, this kind of method which based on the characteristic analysis or decomposition of correlate observation signal matrix is used to calculate the power spectrum density, such as multi-signal classification (MUSIC) method and eigenvector method $(\mathrm{EV})$. This kind of method is suitable for analyzing signal contains the linear spectrum component, especially under the situation of low signalto-noise ratio, the subspace method will show the superiority.

During our research, the calculating method is MUSIC method and the AR model method, the results of them are extremely similar, so we only choose the MUSIC method for mainly calculating method. The general procedure of MUSIC method is as follows[11]:

(1) According to the sample observations $x_{i}$ ( $i=1,2,3 . . n$ ), we get the autocorrelation function $\hat{R}$, assuming $A_{i}$ and $\omega_{i}$ are the power and frequency of the plural sine signals. $\sigma^{2}$ are the white noise variance.

$$
R_{x}(k)=\sum_{i=1}^{M} A_{i} \exp \left(j \omega_{i} k\right)+\sigma^{2} \delta(k)
$$

(2) Through the characteristic decomposition of autocorrelation function $\hat{R}$, we can obtain $P-M+1$ numbers of eigenvalue and the corresponding feature vectors $V_{m+1}, \cdots, V_{p+1}$.

$$
R_{p+1}(k)=\sum_{i=1}^{M}\left(\lambda_{i}+\sigma^{2}\right) V_{i} V_{i}^{H}+\sum_{i=M+1}^{P+1} \sigma^{2} V_{i} V_{i}^{H}
$$

(3) Then, Obtained the power spectrum density.

$$
\hat{P}_{x}(\omega)=\frac{1}{\sum_{i=M+1}^{P+1}\left|a_{i} e^{H}(\omega) V_{i}\right|^{2}}
$$

In equation (3), if $a=1, i=M+1, \cdots, P+1$ the power spectrum estimation method we used is called MUSIC, the equation can be written as equation (4).

$$
\widehat{P}_{x}(\omega)=\frac{1}{e^{H}(\omega)\left(\sum_{i=M+1}^{P+1} V_{i} V_{i}^{H}\right) e(\omega)}
$$

\section{EMPIRICAL RESEARCH}

This paper is aimed to research the hidden circulation fluctuations periodicity of the indices daily return in the Chinese stock markets and foreign stock markets by the MUSIC method of Sub-space of the power spectrum density estimation. From January 1, 2001 to March 31, 2014, the number of the total effective days of the six kinds of indices is more than 3200 , In the process of calculating, we will use the listed days as the unit. The fluctuation curves of the time series of the 6 kinds of Composite Index of stock prices are showing in Fig. 1. The $\mathrm{X}$ axis represents listed days, and the $\mathrm{Y}$ axis number represents the Index daily return.

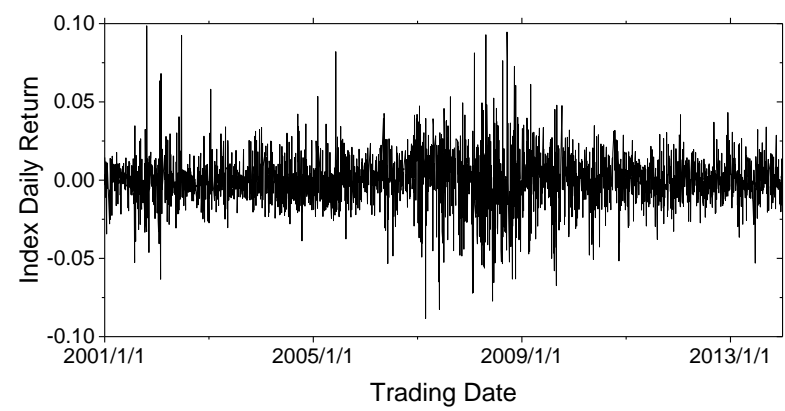

(a) Shanghai Composite Index

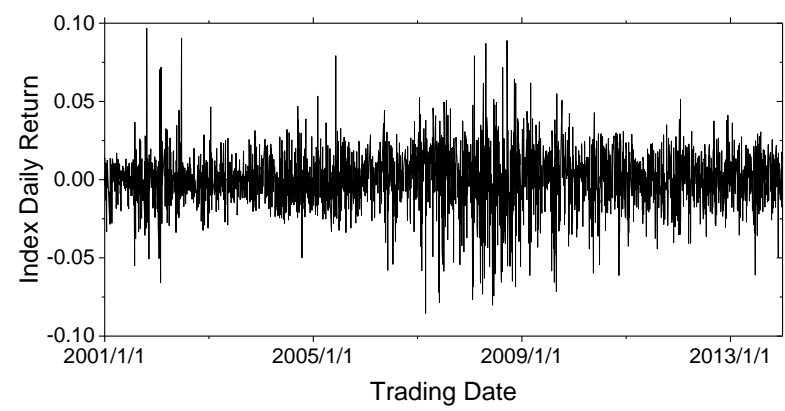

(b) Shenzhen stock composite prices index

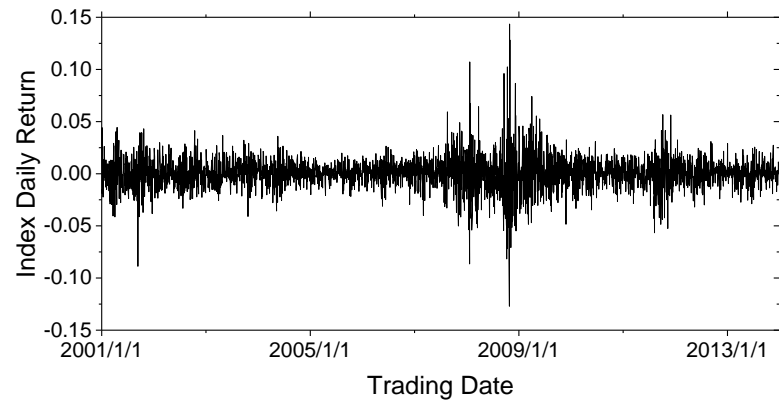

(c) Hang Seng Index

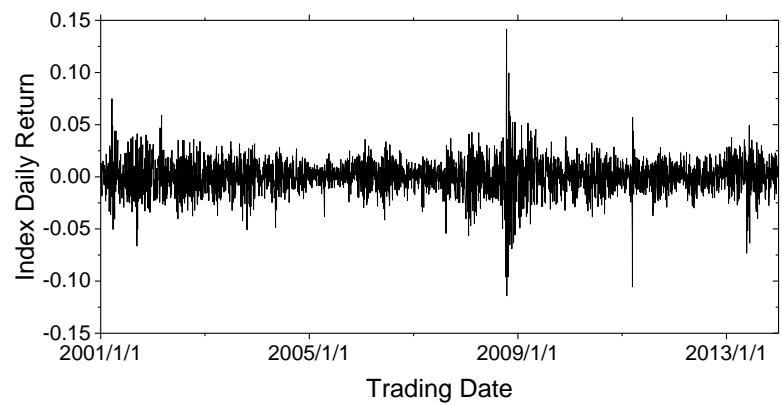

(d) Nikkei 225 index

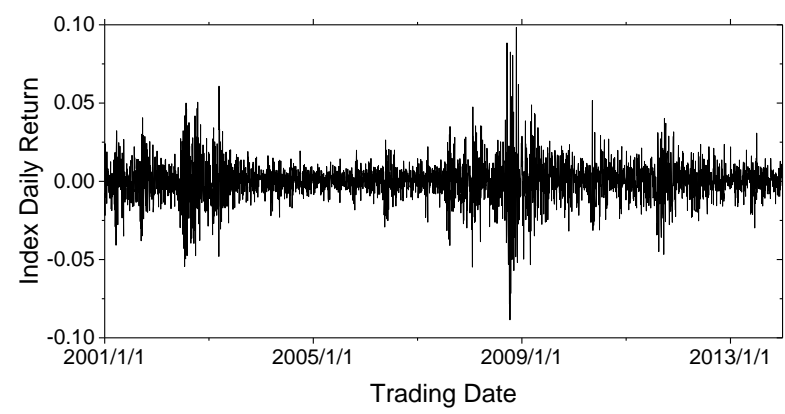

(e) London's FTSE 100 index 


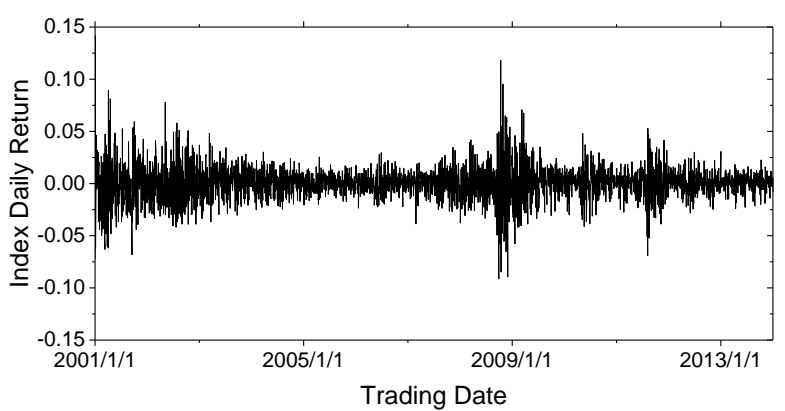

(f) Nasdaq Composite Index

Figure 1. The Index daily return

From Fig. 1, we find that the Hong Kong Stock Exchange, US Stock Markets and Japanese stock markets produce some volatility fluctuations but is not expected to have a lasting impact in 2008. As we known, there was tremendous shake which happening in the second half of 2008. The shake arose in America resulted in a financial crisis over the whole world. Correspondently, the worldwide stock markets have highly functions because of the worldwide crisis.

Through the general research methods, it is difficult to get the hidden circulation fluctuations periodicity. So we chose the spectral analysis method, after the use of the MUSIC method of spectrum analysis, Fig. 2 plots the power spectrum density estimation curves of domestic and foreign

markets.

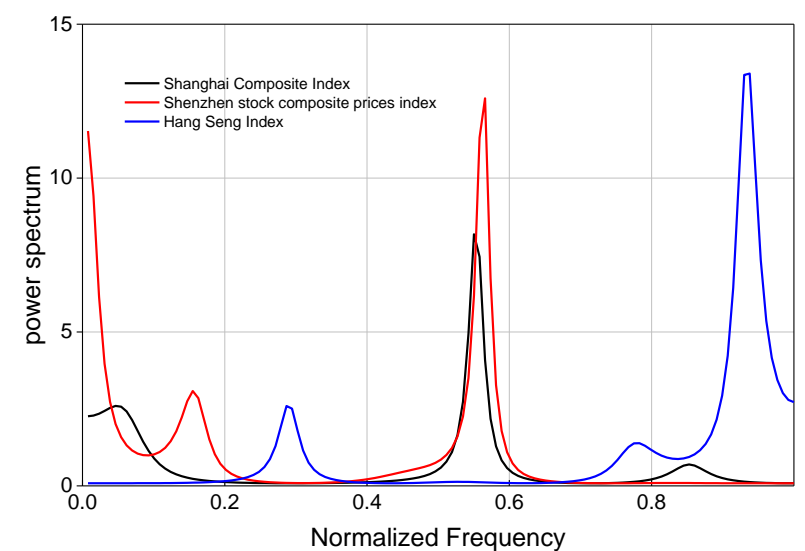

(a) Domestic stock markets

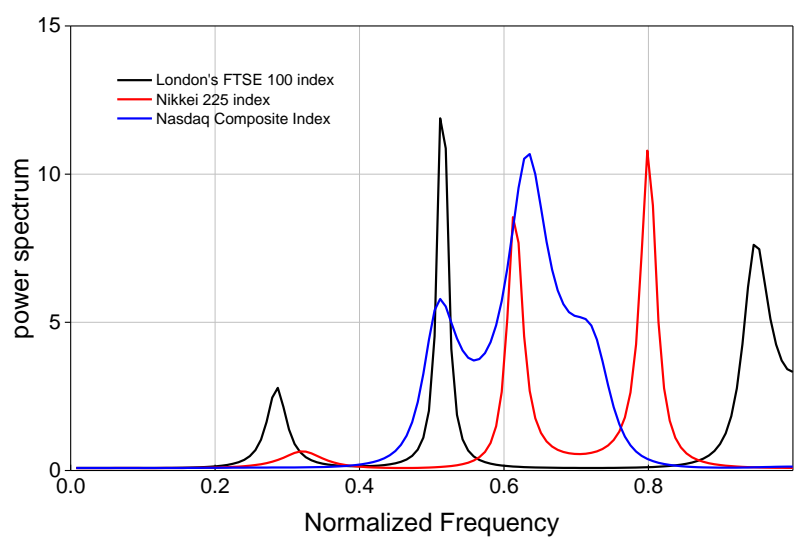

(b) Foreign stock markets

Figure 2. Power spectrum Estimate via MUSIC
In the Fig. 2, the horizontal axis represents the Normalized Frequency $(\times \pi /$ sample $)$, the default sample is 256. It is worth noting that the cycle value is equal to the sample value divide the value of $\pi$ multiply the Normalized Frequency. If the Normalized Frequency is to small, the cycle value will be too large, which has little valuable significance. Thus, we neglect the Normalized Frequency value when it is less than 0.4 .

The Y axis represents the spectral density. It's easy to note that the curves of spectrum analysis of the Shanghai and Shenzhen exchanges have some similar characteristic and the stock markets have certainly hidden periodicity. The different stock markets have different hidden periodicity. We can not find out the relationships of hidden periodicity between the domestic stock markets and foreign stock markets.

After transforming the normalized frequency to cycle value, we can obtain the 2 main orders of periodic components of the daily return of the stock markets as shown in table 1 .

TABLE I. THE 2 MAIN ORDERS OF PERIODIC COMPONENTS OF INDEX DAILY RETURN

\begin{tabular}{|l|c|c|}
\hline Index & Frist & Second \\
\hline Shanghai Composite Index & 95.49 & $147.86^{*}$ \\
\hline Shenzhen stock composite prices index & $143.72^{*}$ & - \\
\hline Hang Seng Index & $86.99^{*}$ & 104.37 \\
\hline Nikkei 225 index & $102.14^{*}$ & 113.30 \\
\hline London's FTSE 100 index & 86.09 & $159.40^{*}$ \\
\hline Nasdaq Composite Index & $128.26^{*}$ & 159.12 \\
\hline
\end{tabular}

We can see that Hang Seng Index has obviously cyclical nature with two closely fluctuation cycle value. The Shanghai Composite Index has a similar cyclical nature to London's FTSE 100 index, but the London's FTSE 100 index has strength fluctuation. We also can get the conclusion that Shanghai has a similar fluctuation to Shenzhen exchanges because they have a closely mainly periodic components around 145. Meanwhile the second periodic components of them either is too small or not exist. These phenomena are also can be observed in fig. 1 . As it is known to all, these composite indices is obtained by calculating a series of stocks price. Thus, it is inevitable to the indices have a more complex fluctuation characteristic. With the research of individual stock, we can select corresponding predict method, such as nonlinear chaotic time series of economic of stock market to forecast stocks movements, which can help us optimize our investment strategies. In addition, if we predict the daily fluctuation period on the basis of researching the prediction of individual stocks' monthly fluctuation, which can further optimize our investment strategies so that we obtain a fruitful economic benefit.

\section{CONCLUSION}

In this article, we bring in the MUSIC method of Sub space of the power spectrum density estimation to analyze the fluctuations of index daily return in domestic stock markets and foreign stock markets. Through our research, we believe that the stock markets have obvious daily hidden periodicity and the curve of spectrum analysis of 
the Shanghai and Shenzhen exchanges are similar. Also the hidden circulation fluctuations periodicity of them is similar to London stock markets. This research also shows that we can get fruitful results when we bring some methods of other academic disciplines to research the time series data of social economy. After we get the daily and monthly hidden periodicity of some stocks and use some other methods to predict the trends of those stocks returns correctly, we can optimize our investment strategies, and maximize our economic benefits. Also, we should pay attention to that the hidden circulation fluctuations periodicity in stock markets may virtually nonexistent because the system of stock market is a very complex nonlinear system. The investors in stock markets have different Individual emotional characteristics and can be influenced by various external factors.

\section{ACKNOWLEDGMENT}

This research is supported by the Fundamental Research Funds for the Central Universities (No. N130323007), the project from Northeastern University at Qinhuangdao (No. XNB201402) and the Social science fund in Hebei province (No. HB14YJ098).

\section{REFERENCES}

[1] George E. P. Box, Gwilym M. Jenkins, Gregory C. Reinsel. "Time Series Analysis Forecasting and Control". 4th Edition. Massachusetts: Wiley publisher, 2008.

[2] Dong-Hua Wang, Yuan-Yuan Suo, Xiao-Wen Yu, et al. "Pricevolume Cross-correlation Analysis of Csi300 Index Futures". Physica A-statistical Mechanics and Its Applications, vol. 392, 2013, pp1172-1179.
[3] Pablo Suarez-garcia, David Gomez-ullate.Scaling, "Stability and Distribution of the High-frequency Returns of the Ibex35 Index". Physica A-statistical Mechanics and Its Applications, vol. 392, 2013, pp1409-1417

[4] Rafal Rak, Stanislaw Drozdz, Jaroslaw Kwapien, et al. "Stock Returns Versus Trading Volume: Is the Correspondence More General?”. Acta Physica Polonica B, vol. 44, 2013, pp2035-2050.

[5] Rosario Bartiromo. "Maximum Entropy Distribution of Stock Price Fluctuations". Physica A-statistical Mechanics and Its Applications, vol, 392, 2013, pp1638-1647.

[6] Brock WA. "Some theory of statistical inference for nonlinear science". Review of Economic studies, vol. 58, 1991, pp697-716.

[7] Gaertner W. "Periodic and A periodic Consumer Behavior. Applied Mathematics and Computation", vol. 22, 1987, pp233254

[8] Y. Y. Ma, X.T. Zhuang, and L.X. Li, " Research on the relationships of the domestic mutual investment of China based on the crossshareholding networks of the listed companies” , Physica A: Statistical Mechanics and its Applications, vol. 390, 2011, pp. 749-759.

[9] Y. Y. Ma, X.T. Zhuang, and L.X. Li, “Complex Networks Based Research on Characteristics of the Shanghai Stock Exchange Richclub,” Journal of Northeastern University(Natural Science), vol. 32, 2011, pp. 400-404.

[10] Li Sun-ming. "Application of modern analytical techniques in the vibration signal”. Beijing: National Defence Industry Press. 2008.

[11] Zhu Xi-jun, Sui Si-lian, etc. "Signal and image processing with the software of MATLAB”. Beijing: Electronic Industry Press. 2009.

[12] Zvonko Kostanjcar, Branko Jeren,Zeljan Juretic. "Impact of Uncertainty in Expected Return Estimation on Stock Price Volatility”. Physica A-statistical Mechanics and Its Applications, vol. 391, 2012, pp563-5571.

[13] Rafal Rak, Stanislaw Drozdz, Jaroslaw Kwapien, et al. "Stock Returns Versus Trading Volume: Is the Correspondence More General?". Acta Physica Polonica B, vol.44, 2013, pp2035-2050. 